\title{
Pulmonary Diseases and Corticosteroids
}

\author{
G.R. Sethi and Kamal Kumar Singhal \\ Department of Pediatrics Maulana Azad Medical College, New Delhi, India
}

\begin{abstract}
Steroids (corticosteroids) are anti-inflammatory drugs. Corticosteroids are used in many pulmonary conditions. Corticosteroids have a proven beneficial role in asthma, croup (Laryngotracheobronchitis), decreasing the risk and severity of respiratory distress syndrome (RDS), allergic bronchopulmonary aspergillosis, interstitial lung disease, hemangioma of trachea, Pulmonary eosinophillic disorders. Role of corticosteroids is controversial in many conditions e.g. idiopathic pulmonary hemosiderosis, bronchiolitis, hypersensitivity pneumonitis, hyperplasia of thymus, bronchiolitis, acute respiratory distress syndrome, aspiration syndromes, atypical pneumonias, laryngeal diphtheria, AIDS, SARS, sarcoidosis, meconium aspiration syndrome (MAS), pulmonary haemorrhage, bronchitis, bronchiolitis obliterans with organizing pneumonia in JRA, histiocytosis, $\alpha-1$ antitrypsin deficiency, bordtella pertusis, pulmonary involvement in histiocytosis. However these are used empirically in many of these conditions despite lack of clear evidence in favour. There is concern about their side effects, especially on growth. Systemic steroids are associated with significant adverse effects. Pulmonary conditions have a strategic advantage that inhaled corticosteroids are useful in many of these. Although inhaled preparations of corticosteroids have been developed to maximise effective treatment of lung diseases characterised by inflammation and reduce the frequency of harmful effects, these have not been eliminated. There are situations where only systemic steroids are useful. Clinicians must weigh the benefits against the potential detrimental effects. It is recommended that standard protocols for use of steroids available in literature should be followed, always keeping a watch on the potential hazards of prolonged use. [Indian J Pediatr 2008; 75 (10) : 1045-1056] E-mail: grsethi56@gmail.com
\end{abstract}

Key words : Steroids; Pulmonary diseases; Interstitial lung disease

Role of steroids is well established in many pulmonary conditions and is instrumental in decreasing morbidity and mortality. In conditions like bronchial asthma these have proved to be the only drug effective in prevention of mortality and improving the quality of life significantly in majority of patients. However there are conditions like bronchiolitis where inflammation is undoubtedly the underlying pathology but steroids have not been proved to be very effective. And in many conditions steroids have been used empirically without evidence. Steroids are used in prevention and treatment of many neonatal conditions.

Present article is review of literature of role of steroids and current status in various pulmonary conditions. Most important condition in pulmonary diseases, which has derived maximum benefit from steroids is probably bronchial asthma.

\section{Respiratory Distress Syndrome (RDS)}

Approximately 1\% of infants develop Respiratory distress syndrome (RDS). Administration of antenatal steroids

Correspondence and Reprint requests : Dr. G.R. Sethi, Professor, Maulana Azad Medical College, New Delhi-110002, India

[Received August 19, 2007; Accepted August 19, 2007] matures the fetal lung, inducing the enzymes for surfactant synthesis and the gene for surfactant proteins. Benefit is maximal in infants delivered within 24 to 168 hours of maternal therapy being started; a smaller benefit is seen in infants whose mothers have received less than 24 hours of treatment.

Antenatal steroids do not increase the risk for infection in pregnancies complicated by premature rupture of membranes.

The recommendations of Royal college of Obstreticians and Gynaecologists and the National Institute of Health in US, conclude that antenatal treatment with corticosteroids should be considered for all women at risk for preterm labour between 24 and 36 hours.

Number of doses of steroids: There have been doubts, however, about the safety of multiple courses of steroids. Several retrospective studies have examined the benefits of repeat antenatal steroids. Many of these indicate that repeat courses decrease the risk and severity of RDS. Several studies, for adverse effects of repeated courses of antenatal steroids in human neonates found decreased birth weight and head circumference, while other studies contradicted these findings. Long-term evaluation suggests that even if birth weight is reduced, this effect is temporary . 


\section{G.R. Sethi and K.K. Singhal}

In a recent randomized, controlled, multicenteric trial, when difference in birth weight was evaluated for those exposed to 0 to 3 additional courses of steroids and those exposed to 4 or more courses of steroids, the birth weight reduction was seen only in the 4 or more group ${ }^{1}$.

The largest clinical trial to date was recently published in the Lancet ${ }^{2}$. Neonates exposed to repeat corticosteroids developed RDS significantly less frequently (33\% versus $41 \%$ ). This group also had less severe lung disease, needed less oxygen therapy, and had a shorter duration of mechanical ventilation. Neonatal weight and head circumference were reduced at birth but were normal at the time of discharge.

Type of steroids: Betamethasone and dexamethasone are the two recommended corticosteroids for the promotion of fetal maturity. Betamethasone (two doses of $12 \mathrm{mg}$, given im, 24 hours apart) rather than dexamethasone (4 doses of $6 \mathrm{mg}$, given im, every 12 hours) is preferred, since betamethasone has been shown to be associated with a lower incidence of cystic PVL, RDS, BPD, Neonatal deaths, impaired neurological status, hearing impairment and other adverse neonatal outcomes.

\section{BRONCHOPULMONARY DYSPLASIA (BPD)}

The cause of BPD in susceptible infants is multifactorial .The immature lung is vulnerable in the stage before alveolar formation begins (23-26 weeks gestation). Factors that increase inflammation in the lungs - such as oxygen toxicity, mechanical ventilation induced trauma from volume and pressure changes, and infection - are associated with the development of BPD. These data suggest that inflammation has an important role in pathogenesis of BPD and that the pharmacological modulation of the inflammatory response may be protective.

The known long and short term side effects of corticosteroids accompany any improvement in pulmonary function. Thus, the routine use of steroid in premature newborn is strongly discouraged, as reflected in strong editorial statements from AAP. However these findings were generally based on data from studies that have used extremely high doses of dexamethasone for prolonged periods of time.

Systematic reviews indicated that treating VLBW infants with corticosteroids, primarily dexamethasone, in the first few weeks of life significantly decreased the duration of ventilator dependence and the incidence of CLD. However, there were significant short term side effects, including hyperglycemia and hypertension and, more important, some evidence of long term side effects, including severe ROP and abnormal neurological examination ${ }^{3}$.

A recently published study, reported use of low dose dexmethasone $(0.89 \mathrm{mg} / \mathrm{kg} /$ day over 10 days $)$ in preterm infants who were ventilator dependent after 1 week of age. The study showed decreased ventilator requirement, improved oxygenation, and greater percentage of successful extubation in the treatment group compared with placebo. But no data on long term neurological outcomes are available for this study ${ }^{4}$.

O'Shea et al, in a follow up, of a randomized, placebo controlled trial of dexamethasone, found that a 42-day tapering course of dexamethasone, does not increase the risk of composite outcome of death or major neurodevelopment impairment ${ }^{5}$.

Route: There is no evidence that inhaled corticosteroids confer net advantages over systemic corticosteroids in the management of ventilator dependent preterm infants.

Timing: Postnatal steroids have been used either for prevention of CLD or to treat it once established. Based on the timing of initiation of the treatment, it can be categorized into 3 broad groups as follows:

(i) Early treatment, during the first 96 hrs after birth; (ii) Moderately early treatment, between postnatal days 7 and 14; (iii) Delayed treatment, given after 3 weeks of age.

The timing of steroid treatment may affect the occurrence of neurodevelopemental complications, although this has not been systemically studied.

Dose and duration: Various studies have used different regimes for postnatal steroid treatment. The most commonly used regimes are summarized in Table 1.

TABle 1. Dose and Duration of Postnatal DexMethasone used in RCTs

\begin{tabular}{|c|c|c|c|}
\hline Regimes & $\begin{array}{l}\text { Early } \\
\text { (First } 96 \text { hrs of life) }\end{array}$ & $\begin{array}{l}\text { Moderately early } \\
\text { (Between } 7 \text { \& } 14 \text { days of life) }\end{array}$ & $\begin{array}{l}\text { Late } \\
\text { (After } 3 \text { weeks of age) }\end{array}$ \\
\hline Initial dose & $0.5 \mathrm{mg} / \mathrm{kg} / \mathrm{d}$ for 3 days & $\begin{array}{l}0.5 \mathrm{mg} / \mathrm{kg} / \mathrm{d} \text { through out study } \\
\text { period (or) } 0.5 \mathrm{mg} / \mathrm{kg} / \mathrm{d} \text { as a } \\
\text { pulse dose for } 3 \text { days every } 10 \text { days } \\
\text { until } 36 \text { w } \mathrm{ks} \text { PMA }\end{array}$ & $\begin{array}{l}0.5 \text { to } 1 \mathrm{mg} / \mathrm{kg} / \mathrm{d} \text { through } \\
\text { out the study period }\end{array}$ \\
\hline Tapering & $\begin{array}{l}0.25 \mathrm{mg} / \mathrm{kg} / \mathrm{d} \text { for } 3 \text { days } \\
0.125 \mathrm{mg} / \mathrm{kg} / \mathrm{d} \text { for } 3 \text { days } \\
0.05 \mathrm{mg} / \mathrm{kg} / \mathrm{d} \text { for } 3 \text { days }\end{array}$ & $\begin{array}{l}\text { Either maintained in same dose } \\
\text { (or) tapered in different ways }\end{array}$ & Tapered every 3 days in different ways \\
\hline Duration* & 12 days (1-28 days) & 7 days (2-42 days) & 3 days to 3 week \\
\hline
\end{tabular}

*mode (range); PMA: postmenstrual age. 


\section{Pulmonary Diseases and Corticosteroids}

Type of steroids: Recent arguments have questioned the use of dexamethasone in the steroid trials and the possible use of other steroids.

Andre et al in a study of preterm infants at risk of chronic lung disease found that those treated with methylprednisolone had higher rate of body weight gain and a lower incidence of glucose intolerance and cystic PVL compared with cases treated with dexamethasone ${ }^{6}$.

In one study a shorter course and lower dose of betamathasone was nearly as effective as Dexamethasone in weaning ventilatory support, without the undesirable, short term, side effects of dexamethasone. However more trials are required to study the role of betamethasone.

A study by Watterberg comparing placebo with hydrocortisone, in preterm infants weighing less than $1 \mathrm{~kg}$ and mechanically ventilated, showed no significant difference in survival rates between the two groups ${ }^{7}$. In conclusion, the role of postnatal corticosteroids in chronic lung disease is still controversial. It might be prudent to reserve the use of late (After 3 weeks of age) corticosteroids to infants who cannot be weaned from mechanical ventilation, and to minimize the dose and duration of any course of treatment. Role of steroids, other than dexamethasone, need to be studied.

\section{ASTHMA}

Medications to treat asthma can be classified as controllers or relievers. Controllers are medications taken daily on a long-term basis to keep asthma under clinical control chiefly through their anti-inflammatory effects. Relievers are medications used on as-needed bases that act quickly to reverse bronchoconstriction and relieve its symptoms. In this manuscript we will be emphasizing only the role of steroids in asthma, and its current status and not a detailed management of asthma. However the recommendations for use of steroids are based on published concensus protocols, mainly the one suggested by Global Initiative for Asthma. ${ }^{8}$

Corticosteroids are one of the most important component of therapy for asthma; however, the corticosteroid doses, delivery and agents used in the therapy of pediatric asthma vary considerably. Corticosteroids can be administered in different waysinhaled, orally, or by injection. Inhaled therapy is the cornerstone of asthma treatment for children of all ages. The major advantage of inhaled therapy is that drugs are delivered directly into the airways, producing higher local concentrations with significantly less risk of systemic side effects.

Role of steroids in management of persistent asthma: In preschool aged children with persistent asthma, these agents can be effectively delivered, by both MDI via holding chamber and by nebulizer, with the efficacy in decreasing asthmatic symptoms. A metered-dose inhaler (MDI) with spacer is preferable to nebulized therapy. If commercially produced spacers are not available or feasible, homemade spacers have been found to be equally effective (for example, one made from a 500/1000 $\mathrm{ml}$ plastic bottle).

Inhaled glucocorticoids in persistent asthma: Inhaled glucocorticosteroids are currently the most effective antiinflammatory medications for the treatment of persistent asthma. Studies have demonstrated their efficacy in reducing asthma symptoms, improving quality of life, improving lung function, decreasing airway hyperresponsiveness, controlling airway inflammation, reducing frequency and severity of exacerbations, and reducing asthma mortality. However, they do not cure asthma, and when they are discontinued deterioration of clinical control follows within weeks to months in a proportion of patients.

Children older than 5 years. Dose-response studies and dose titration studies in children, demonstrate marked and rapid clinical improvements in symptoms and lung function at low doses of inhaled glucocorticosteroids (e.g., 100-200 $\mu$ g budesonide daily) and mild disease is well controlled by such doses in the majority of patients. Some patients require higher doses (400 $\mu \mathrm{gm} /$ day) to achieve optimal asthma control and effective protection against exercise-induced asthma.

Children 5 years and younger. Treatment with inhaled glucocorticosteroids in children 5 years and younger with asthma generally produces similar clinical effects as in older children, but dose-response relationships have been less well studied. With use of a spacer device, daily doses $\leq 400 \mu \mathrm{gm}$ of budesonide or equivalent result in near maximum benefits in the majority of patients.

Table 2 Lists approximately equipotent doses of different inhaled glucocorticosteroids administered via different inhalation devices.

TABLE 2. Estimated Equipotent Daily Doses of Inhaled Glucocorticosteroids for Children

\begin{tabular}{lccc}
\hline Drug Low & $\begin{array}{c}\text { Daily Dose } \\
(\mu \mathbf{g m})\end{array}$ & $\begin{array}{c}\text { Medium Daily } \\
\text { Dose }(\mu \mathbf{g m})\end{array}$ & $\begin{array}{c}\text { High Daily } \\
\text { Dose }(\mu \mathbf{g m})\end{array}$ \\
\hline $\begin{array}{l}\text { Beclomethasone } \\
\text { dipropionate }\end{array}$ & $100-200$ & $>200-400$ & $>400$ \\
$\begin{array}{l}\text { Budesonide* } \\
\text { Budesonide-Neb }\end{array}$ & $100-200$ & $>200-400$ & $>400$ \\
$\begin{array}{l}\text { Ciclesonide* } \\
\text { Flunisolide }\end{array}$ & $80-160$ & $>500-1000$ & $>1000$ \\
$\begin{array}{l}\text { Fluticasone } \\
\begin{array}{l}\text { Mometasone } \\
\text { furoate* }\end{array}\end{array}$ & $500-750$ & $>750-320$ & $>320$ \\
$\begin{array}{l}\text { Triamcinolone } \\
\text { acetonide }\end{array}$ & $100-200$ & $>200-500$ & $>500$ \\
\hline
\end{tabular}




\section{G.R. Sethi and K.K. Singhal}

Patients considered for high daily doses except for short periods should be referred to a specialist for assessment to consider alternative combinations of controllers.

*Approved for once daily dosing in mild patients.

Notes

The most important determinant of appropriate dosing is the clinician's judgment of the patient's response to therapy, in terms of clinical control. Once control of asthma is achieved, the dose of medication should be carefully titrated to the minimum dose required to maintain control, thus reducing the potential for adverse effects.

Side effects: Glucocorticosteroid-induced changes in growth rate during the first year of treatment appear to be temporary. Oral or systemic glucocorticosteroid use increases the risk of fracture, but there are no adverse effects of inhaled glucocorticosteroid treatment on bone mineral density. Inhaled glucocorticosteroid doses of less than $200 \mu \mathrm{gm}$ budesonide or equivalent daily is normally not associated with any significant suppression of the HPA axis in children. The use of inhaled glucocorticosteroids is not associated with an increased incidence of lower respiratory tract infections, including tuberculosis, central nervous system effects, cataract development, clinical thrush and dental caries in children.

Leukotriene modifiers and Long-acting inhaled $\boldsymbol{\beta}_{2}$ agonists: Leukotriene modifiers alone provide clinical benefit, in children older than 5 years at all levels of severity, but generally less than that of low-dose inhaled glucocorticosteroids. As add-on treatment in children whose asthma is insufficiently controlled by low doses of inhaled glucocorticosteroids, leukotriene modifiers provide moderate clinical improvements, including a significant reduction in exacerbations.

Long-acting inhaled $\beta_{2}$-agonists have mainly been studied in children older than 5 years as add-on therapy for patients whose asthma is not controlled on low to high doses of inhaled glucocorticosteroids as a steroid sparing option. Combination products containing an inhaled glucocorticosteroid and a longacting inhaled $\beta_{2}$-agonists are preferred to long-acting inhaled $\beta_{2}$-agonists and inhaled glucocorticosteroids administered by separate inhalers. Fixed combination inhalers ensure that the long-acting $\beta_{2}$-agonists is always accompanied by a glucocorticosteroid.

The effect of long-acting inhaled $\beta_{2}$-agonists has not yet been adequately studied in children 5 years or younger.

Combination therapy with budesonide and formoterol used both as maintenance and rescue has been shown to reduce asthma exacerbations in children ages 4 years and older with moderate to severe asthma .

Systemic glucocorticosteroids in persistent asthma: Oral glucocorticosteroids in children with persistent asthma should be restricted to the treatment of acute severe exacerbations, because of the side effects of prolonged use. However a minority of chronic severe asthma may require low dose systemic steroids in addition to ICS.

Treatment steps for achieving control: The patient's current level of asthma control and current treatment determines the selection of pharmacologic treatment. For example, if asthma is not controlled on the current treatment regimen, treatment should be stepped up until control is achieved. However no stepping up should be done without giving due care to check dose, compliance, technique of breathing, proper use of devices and co morbid conditions.

If control has been maintained for at least three months, treatment can be stepped down with the aim of establishing the lowest step and dose of treatment that maintains control.

When inhaled glucocorticosteroids alone are being used, a reduction in dose should be attempted at 3 month intervals and when control is achieved at a lowdose, treatment may be switched to once-daily dosing. When asthma is controlled with a combination of inhaled glucocorticosteroid and long-acting $\beta_{2^{-}}$ agonists, begin by reducing the dose of inhaled glucocorticosteroid until a low-dose is reached, when the long acting $\beta_{2}$-agonists may be stopped. Controller treatment may be stopped if the patient's asthma remains controlled on the lowest dose of controller and no recurrence of symptoms occurs for one year.

Difficult to treat asthma: Patients who do not reach an acceptable level of control at Step 4 (reliever medication plus two or more controllers) can be considered to have difficult-to-treat asthma. Dose optimization should be pursued, by stepping down to a dose that maintains the maximal level of control. Because very few patients are completely resistant to glucocorticosteroids, these medications remain a mainstay of therapy for difficultto-treat asthma. Additional diagnostic and therapeutic options that can be considered are 1. cyclosporin in persistent eosinophillic inflammation 2. azithromycin in persistent neutrophilic inflammation 3. continuous subcutaneous terbutaline by pump in airway reactivity without inflammation

Role of steroids in the management of asthma exacerbations

Systemic glucocorticosteroids. Systemic glucocorticosteroids speed resolution of exacerbations and should be utilized in the all but the mildest exacerbations, especially if:

- The initial rapid-acting inhaled $\beta_{2}$-agonists therapy fails to achieve lasting improvement

- The exacerbation develops even though the patient was already taking inhaled glucocorticosteroids

Indian Journal of Pediatrics, Volume 75-October, 2008 


\section{Pulmonary Diseases and Corticosteroids}

- Previous exacerbations required oral glucocorticosteroids.

Oral glucocorticosteroids are usually as effective as those administered intravenously and are preferred because this route of delivery is less invasive and less expensive. An oral glucocorticosteroid dose of $1 \mathrm{mg} / \mathrm{kg}$ daily is adequate for treatment of exacerbations in children with mild persistent asthma. A 7-day course in adults has been found to be as effective as a 14-day course, and a 3- to 5-day course in children is usually considered appropriate.

Inhaled glucocorticosteroids. Cochrane review concluded that there is insufficient evidence that ICS therapy provides additional benefit when used in combination with standard CS therapy upon emergency department discharge for acute asthma. Further research is needed to clarify whether ICS therapy should be employed in acute asthma treatment in the emergency department or following emergency department discharge. ${ }^{9}$

For detailed discussion on use of steroids reader may refer to current consensus guidelines published in literature.

\section{CROUP}

Croup (Laryngotracheobronchitis) is the most common form of airway obstruction in children between the ages of 6 months and 6 years, peaking between the ages of 1 and 2 years. The symptoms are thought to occur as a result of edema of the larynx and trachea, which has been triggered by a recent viral infection.

The standard management of croup includes mist treatment (that is, treatment with humidified air), although there is little evidence of its effectiveness. Racemic adrenaline (epinephrine), or L-adrenaline, has been shown to provide temporary relief to patients with croup but is not thought to have long-term benefits .

The therapeutic role of corticosteroids is no longer in doubt. The cumulative evidence strongly supports their use in children and moderate to severe symptoms. Cochrane review ${ }^{9}$ concluded that glucocorticoids bring about clinical improvement in children with croup within six hours. They found that nebulised budesonide or systemic dexamethasone, given either orally or intramuscularly, is effective in treating croup. The use of glucocorticoids is associated with a lower rate of epinephrine use, fewer return visits and/or (re) admission and shorter time spent in hospital. Most clinical studies have reported effects of a single dose of corticosteroid by any route. In children with poor response or worsening of symptoms, according to literature on intensive care management of children with croup, multiple doses are often routinely administered ; these also protect against postextubation airway edema. Outside the pediatric intensive care unit, there is no good quality evidence to guide this decision.

A recent randomized equivalence trial concluded that a single oral dose of prednisolone $(1 \mathrm{mg} / \mathrm{kg})$ is less effective than a single oral dose of dexamethasone $(0.15 \mathrm{mg} / \mathrm{kg})$ in reducing unscheduled re-presentation to medical care in children with mild to moderate croup. ${ }^{10}$

\section{BRONCHIOLITIS}

Bronchiolitis is a worldwide common lower respiratory tract infection in infants. The treatment for most infants with bronchiolitis is usually supportive, including oxygen, hydration, and antipyretics. Treatment effects from bronchodilators have been examined in detail. The use of bronchodilator therapy remains controversial.

Theoretically, anti-inflammatory drugs should help, particularly in the early phase of bronchiolitis, as inflammation of the bronchioles is the main pathological feature. Initial studies of the treatment of bronchiolitis with corticosteroids suggested that these drugs might favorably influence mortality and morbidity. However Cochrane (2004) meta analysis concluded that systemic glucocorticoids do not appear to have a clinically significant effect on the course of acute bronchiolitis in infants and young children. ${ }^{11} \mathrm{~A}$ recent Cochrane review (2007) concluded that there is a limited data on the effect of inhaled corticosteroids during acute bronchiolitis on the prevention of post bronchiolitic wheezing. ${ }^{12}$

The most recent clinical practice guidelines from AAP do not recommend corticosteroids for routine management of acute bronchiolitis, based on randomized controlled trials with limitations and a preponderance of risk over benefits.

The controversy has remained alive still with publication of two randomized controlled trials in past one year giving out contradictory results ${ }^{13,14}$. In a randomized, double blind, placebo controlled trial, Teeratakulpisarn et al concluded that a single injection of dexamethasone yielded a significant clinical benefit for the treatment of previously healthy, young children hospitalized with acute bronchiolitis. ${ }^{13}$ In the recent, double blind, randomized trial, comparing single dose of oral dexamethasone $(1 \mathrm{mg} / \mathrm{kg})$ with placebo in 600 children (age range 2 to 12 months) with a first episode of wheezing diagnosed, in emergency department, as moderate to severe bronchiolitis, Corneli et al, concluded that it did not significantly alter rate of hospital admission, the respiratory status after 4 hours of observation, or later outcomes. ${ }^{14}$

Studies available vary widely in patient populations, 


\section{G.R. Sethi and K.K. Singhal}

interventions and outcome variables. Therefore till date the utility of steroids in bronchiolitis has not been proven beyond doubt.

\section{INERSTITIAL LUNG DISEASE (ILD)}

The multiple possible diagnostic entities and lack of randomized clinical trials make offering specific recommendations regarding treatment of children's interstitial lung disease (ILD) difficult. If the process is secondary to an underlying condition, patients should be treated for the underlying disease.

The principles of treatment include meticulous attention to growth and nutrition, immunizations (including influenza and pneumococcal prophylaxis), and treatment of secondary infections

In, general corticosteroids remain the treatment of choice for most patients with ILD on the presumption that suppression of inflammation might be beneficial. Hypersensitivity pneumonitis is the most treatable condition among ILD. Other steroid-responsive conditions include nonspecific interstitial pneumonitis (NSIP), desquamative interstitial pneumonitis (DIP), lymphocytic interstitial pneumonitis (LIP), cryptogenic organizing pneumonia (COP), eosinophilic pneumonia syndromes, sarcoidosis, pulmonary hemosiderosis, and ILD associated with connective tissue disease. In fibrotic lung diseases such as idiopathic pulmonary fibrosis (IPF) inflammation is more limited, whereas fibrogenesis appears to be the primary disease process. Consequently, anti-inflammatory therapy has not been proven to be effective in Idiopathic pulmonary fibrosis (IPF) and antifibrotic therapies are currently being developed.

The elimination of alveolitis is the only therapeutic treatment for idiopathic diffuse interstitial lung disease (DILD), since no antifibrotic drugs of proven efficacy are currently available. The drugs used are corticosteroids and immunosuppressants. Indications and duration of treatment vary depending on the form of ILD. Intravenous pulse steroid therapy is becoming a common alternative to oral therapy, because it works at least as well or better, and it is associated with fewer side effects.

\section{STEROID TREATMENT OF DIFFERENT FORMS OF ILD}

Idiopathic Pulmonary Fibrosis (IPF): The only therapeutic strategy shown to be useful in slightly increasing survival is combined treatment with corticosteroids and azathioprine. The ATS/ERS have established guidelines concerning the therapeutic
Table 3. Treatment of Idiopathic Pulmonary Fibrosis

Corticostiroids (prednisolone or eqivalent)

$0.5 \mathrm{mg} / \mathrm{kg}$ weight/day, oral, 4 weeks

$0.25 \mathrm{mg} / \mathrm{kg}$ weight/day, oral, 8 weeks

Reduce dose to $0.125 \mathrm{mg} / \mathrm{kg}$ weight/day

or $0.25 \mathrm{mg} / \mathrm{kg}$ weight/alternate days

Combine with azathioprine or cyclophosphamide

Azathioprine

2-3 mg/kg weight/day

Maximum dose:150 mg/day

Initial dose: $25-50 \mathrm{mg} /$ day

Increase the dose by 25- $50 \mathrm{mg}$ every 1-2 weeks

Cyclophosphamide

$2 \mathrm{mg} / \mathrm{kg}$ weight/day

Maximum dose:150 mg/day

Initial dose: $25-50 \mathrm{mg} / \mathrm{day}$

Increase the dose by 25- 50 mg every 1-2 weeks

regimen that should be used (Table 3$)^{15}$.The duration of treatment depends on the course of the disease, but maintaining the initial regimen for at least 6 months is recommended. If the patient's condition is found to have improved or stabilized, the same regimen should be continued. If the patient's condition has deteriorated after 6 to 12 months of treatment, the possibility of a lung transplant should be considered.

The recommended dose of methylprednisolone is 30 $\mathrm{mg} / \mathrm{kg}$, administered intravenously over one hour daily, for 3 consecutive days, and repeated monthly.

Nonspecific Interstitial Pneumonia (NSIP): Oral corticosteroids (prednisone or equivalent), at a dosage of $1 \mathrm{mg} / \mathrm{kg}$ of weight (maximum $80 \mathrm{mg}$ ), for one month. The dose is then reduced by $10 \mathrm{mg}$ every 15 days until reaching $20 \mathrm{mg}$. This dose is maintained for 2 weeks, after which it is reduced to between $5 \mathrm{mg}$ and $10 \mathrm{mg}$ administered on alternate days, a regimen that is maintained until clinical resolution and stabilization of respiratory function parameters is achieved. If the patient does not respond to corticosteroid treatment, azathioprine is added using the dosage regimen recommended for IPF.

Dosage regimen recommended for NSIP are also used for treating desquamative interstitial pneumonitis (DIP), lymphocytic interstitial pneumonitis (LIP)

Cryptogenic Organizing Pneumonia: Oral corticosteroids at the dosage regimen used for NSIP. If the patient does not respond to treatment or requires prolonged treatment with corticosteroids, azathioprine at the same dosage regimen used in IPF can be added. However, there is no experience with the effect of this drug in COP.

Acute Interstitial Pneumonia: Although no controlled trials have been carried out, treatment with high doses of corticosteroids (100-250 mg/day of intravenous 


\section{Pulmonary Diseases and Corticosteroids}

methylprednisolone) has been shown to be effective in the exudative stage of the disease .

Sarcoidosis: Sarcoidosis is a common, multi system granulomatous disorder. Lungs are frequently involved and can often progress to pulmonary fibrosis. Pulmonary sarcoidosis is characterised by a course that is unpredictable, with some patients showing spontaneous resolution while others progress to chronic lung disease including lung fibrosis. It is not possible currently to identify those patients who are in the latter group. Although corticosteroid therapy has been widely observed to alleviate symptoms and improve disease manifestations, efficacy of corticosteroid therapy for lung disease remains controversial. Cochrane review concluded that patients with stage 1 disease (bilateral hilar lymphadenopathy alone) do not need treatment with oral steroids, but that those with interstitial lung disease (stages 2 and 3) may show an improvement in CXR and in global scores when treated with oral steroids. There is little evidence that treatment with oral steroids has a beneficial effect on lung function. The available data provides little guidance for the management of this disease after 2 years. There is limited evidence that oral steroids alter disease progression and early treatment alters progression of the disease. ${ }^{16}$ There is little evidence for the efficacy of inhaled steroids. It is well recognized that sarcoidosis is also commonly an endobronchial disease and can cause a troublesome cough. Inhaled steroids should, in theory, be beneficial for this symptom, but this is yet to be established in randomised trials. The optimal dose and length of corticosteroid therapy have not been evaluated in randomized, controlled trials. Treatment protocols for children vary modestly, but most suggest initial treatment with $1 \mathrm{mg} /$ $\mathrm{kg} /$ day of prednisolone. Gradual reduction to the maintenance dose should begin when symptoms or manifestations have improved, typically after 4 to 8 weeks. Thus maintenance dose is the lowest dose that controls symptoms, often in the range of 10 to $15 \mathrm{mg}$ given daily or, if possible, every other day. Total duration of therapy is typically at least 6 months. Relapse that occurs during tapering or following completion of therapy may require reinitiating of treatment using similar regimen.

Collagen Diseases: Some patients with collagen diseases (rheumatoid arthritis, systemic sclerosis, dermatomyositis/ polymyositis, systemic lupus erythematosus, Sjögren's syndrome, connective tissue disease) may present DILD during the course of their illness.

(a) Dermatomyositis: Corticosteroids are the mainstay for treating pulmonary disease in dermatomyositis, particularly with an acute onset and early chronic fibrosis. Cochrane review concluded that corticosteroids are the principle treatment but due to side effects, there is a need for additional treatment from drugs that suppress the immune system (immunosuppressants) or modify it (immunomodulatory therapies) to improve disease response. ${ }^{17}$

(b) Systematic lupus erythematosus: Intravenous methylprednisolone pulse therapy, $30 \mathrm{mg} /$ $\mathrm{kg}$ (maximum of $1 \mathrm{mg} / \mathrm{kg}$ ) for 3 day, and / or immunosuppressive drugs or plasmapheresis may be effective in containing and treating with SLE pulmonary complication

(c) Juvenile Rheumatoid Arthritis: Bronch-iolitis obliterans with organizing pneumonia in JRA may respond favorably to steroid therapy.

(d) Rheumatic Pneumonia: Rapid clearance of Rheumatic pneumonitis following treatment with steroids has been described

Radiotherapy induced pneumonitis: Corticosteroids at the dosage used for NSIP are indicated for pneumonitis. However, corticoid therapy has not been shown to be effective in the treatment of fibrosis.

Drug induced pneumonitis: When the clinical, radiological, and/or lung function abnormalities persist, despite stopping the suspected drug, treatment with corticosteroids is indicated (see treatment of NSIP).

Histiocytosis: The use of steroid and other immunosuppressive regimens remain unproven, in pulmonary involvement in histiocytosis, although prospective trials are ongoing.

Pulmonary Esinophilia: Steroids have been tried in almost all pulmonary eosinophilic disorders. The etiologies of pulmonary eosinophilic disorders is given in table 4.

Oral corticosteroids are started at a dosage of $0.5-1 \mathrm{mg} /$ $\mathrm{kg}$ per day. The dose is gradually reduced depending on the course of the disease. Treatment should

Table 4. Etiology of Pulmonary Esinophilias Idiopathic

Chronic esinophilic pneumonia

Acute esinophilic pneumonia

Hyperesinophilic syndrome

Known etiology

Allergic bronchopulmonary aspergillosis Helminths

Drugs

Associated with systemic vasculitis

Churg-Strauss syndrome

Wegener's granulomatosis

Panarteritis nodosa 


\section{G.R. Sethi and K.K. Singhal}

generally be maintained for 6 to 12 months, and a low dose is occasionally required for several years. The treatment for acute eosinophilic pneumonia is high doses of methylprednisolone (1-2 mg/kg every 6 hours for 2 to 3 days), followed by a lower dose $(0.5 \mathrm{mg} / \mathrm{kg}$ for 2 weeks) and gradual tapering of the dose until treatment is completed.

Idiopathic pulmonary hemosiderosis (IPH): Generally, patients who are symptomatic are treated with high doses of corticosteroids. Anecdotally, use of this therapy is associated with cessation of the bleeding. There is no convincing evidence that maintenance corticosteroid therapy prevents recurrent bleeding episodes.

Patient who experience repeated life-threatening episodes, or who experience progression of pulmonary dysfunction over time, may be candidates for immunosuppressive drug therapy. If patient is doing well all attempt should be made to discontinue all therapy within one year, but reinstitution of pharmacological intervention may be required. Inhaled steroids have also been used but no trials been conducted.

In a recent retrospective case series of 26 patients of IPH, Kabra SK et al, concluded that treatment with prednisolone and hydroxy chloroquine followed by inhaled corticosteroids might improve survival in infants with IPH. ${ }^{18}$

Allergic angitis and Granulomatosis: Approximately $50 \%$ of the affected people die within 3 months of the onset of vasculitis; however those treated with corticosteroids have a mean survival time of 9 years. Therapy is usually continued or as long as 1 year.

\section{CYSTIC FIBROSIS}

The mainstay of treatment of cystic fibrosis (CF) is attention to nutrition, vigorous airway clearance, aggressive infection control, and anti-inflammatory therapy.

It has been recognized that the inflammatory process itself may contribute to lung injury. The inflammatory process occurs early in life, in infants as young as four weeks. Even infants without clinically obvious lung disease still have evidence of airway infection and inflammation. Thus anti-inflammatory therapy is useful in patients with CF, particularly those who are relatively young and have only mild disease.

The beneficial effects of anti-inflammatory treatment with inhaled corticosteroids may be greatest among young children with mild disease, in whom fixed structural damage to the lungs has not yet occurred. They may also be of benefit in those with bronchial hyperreactivity, allergic bronchopulmonary spergillosis or chronic Pseudomonas aeruginosa infection.

Although oral corticosteroids are effective in slowing the decline of the FEV1 the complications of steroids in CF make them unsuitable for long term use. Thus treatment regimens need to be adjusted to find the lowest effective dose and the shortest duration of therapy in order to minimize the risk of permanent growth impairment, especially in boys.

Cochrane meta analysis concluded that oral corticosteroids at a prednisolone equivalent dose of $2 \mathrm{mg} / \mathrm{kg}$ on alternate days is effective but should not be used due to the high risk of occurrence of important side effects. A dose of $1 \mathrm{mg} / \mathrm{kg}$ on alternate days might be considered for upto 24 months but close attention to the occurrence of adverse effects is warranted. ${ }^{19}$

Recently, the use of inhaled corticosteroids has increased as well, although it is difficult to discern whether the therapeutic intent is control of asthmatic symptoms or anti-inflammatory therapy directed at the disease process itself. Some studies suggest that such treatment is beneficial, but one small trial was halted when several of the treated patients had new acquisition of P.aeruginosa. ${ }^{20}$

Recently Cochrane review found no evidence, to support the practice of prescribing inhaled steroids in CF. They could not confirm that inhaled steroids are beneficial or that they are not associated with harmful effects. Thus while it is possible that a proportion of people with CF with wheezing may respond to inhaled corticosteroids, existing evidence does not support their routine use in all people with CF. Trials of starting inhaled corticosteroids are becoming increasingly difficult in patient population populations, where a high proportion of people are already taking inhaled corticosteroids. ${ }^{20}$

In view of this, a multicenter randomized double blind placebo controlled trial assessed the safety of withdrawal of inhaled corticosteroids (ICS). They concluded that stopping ICS is not harmful for patients with $\mathrm{CF}$ who have been taking them for some years. It is likely that the majority of patients taking them no longer need to do so. They advocated that prescribing practice of ICS in a CF patient becomes more like that of an asthmatic, justification is needed to start them, reassessment is necessary to see whether they are having an effect and consideration is always given to reduce the dose or stopping them altogether. ${ }^{21}$

\section{ALLERGIC BRONCHOPULONARY ASPERGILLOSIS (ABPA)}

Most cases of ABPA require treatment with corticosteroids, and the treatment of choice is prednisolone. Steroid therapy 


\section{Pulmonary Diseases and Corticosteroids}

rapidly clears hypereosinophilic infiltrates and the associated symptoms, although it is less effective at treating mucous impaction. The usual starting dose is 0.5 $\mathrm{mg} / \mathrm{kg} /$ day, taken each morning, and this dose is maintained for 2 to 4 weeks while following the patient clinically and checking the chest radiograph for resolution of the acute process. After this induction treatment, the dose of prednisolone should be reduced to $0.5 \mathrm{mg} / \mathrm{kg}$ given on alternate day.

Following resolution of acute process, the dose of prednisolone should be reduced over 1 to 3 months. Chronic treatment with corticosteroids is controversial, especially in adults, because only a minority of patients with ABPA, are at risk of chronic lung disease.

The relationship between acute episodes and lung damage is unclear, and the precise dose of prednisolone is not certain, since acute exacerbation may continue while the patients are on low doses of steroids. However, children with ABPA usually have cystic fibrosis and may need treatment with long term corticosteroids to prevent long term lung damage. Therefore maintain therapy with a dose of $0.5 \mathrm{mg} / \mathrm{kg}$ on alternate day for 3 months and then, after 3 months, the dose of prednisolone is tapered over a further 3 months while checking the chest radiograph and the serum IgE level for evidence of relapse. Initially the serum IgE level should be checked at every visit and if the level increases by two fold or more, the steroid dose should be increased.

Recently, Itraconazole has been used as an adjunctive therapy in an attempt to reduce the doses of steroids that are required. A more recent randomized controlled trial showed that treatment of stable ABPA in adults with itraconazole resulted in significant reduction in sputum eosinophil count, sputum eosinophilic cationic protein levels, serum IgE concentrations, Aspergillus-specific IgG, and episodes of exacerbation requiring systemic steroids ${ }^{22}$. In children with ABPA, itraconazole has been used in a dose of 10 $\mathrm{mg} / \mathrm{kg} /$ day.

\section{TUBERCULOSIS}

Corticosteroid administration is beneficial in the management of children when the host inflammatory reaction contributes significantly to tissue damage or impaired function. While role in managing CNS tuberculosis is less controversial, steroids have very little role in pulmonary conditions. Steroids have been used in military TB, effusions, and compressive lymphadenopathy. The two interventions useful in reducing pulmonary injury, in military tuberculosis, are specific antimicrobial therapy and non-specific antiinflammatory therapy (corticosteroids). Though ATT, as such, cannot be expected to reverse the capillary damage, the supportive therapy with ventilation and steroids may be life saving. Steroid therapy appears to be effective in reducing systemic toxicity and intensity of inflammatory exudative pulmonary response ${ }^{23}$. Corticosteroid administration may significantly reduce the compression of the tracheobronchial tree caused by hilar lymphadenopathy, resulting in collapse or obstructive emphysema. Steroids have been used in pleural effusion, and pericardial effusion as well.

Corticosteroids increase the rate at which tuberculous effusion resolve and fever return to normal, but the definitive proof of their value on eventual ventillatory function has not been shown. If steroids are to be used to help alleviate dyspnea, concomitant antituberculous therapy is essential. A recent study assessed adjunct therapy with corticosteroids or paracentesis for treatment of tuberculous pleural effusion. Patients were divided into 3 groups. All groups were treated with antituberculosis drug for 6 months; in group 2, prednisolone, $30 \mathrm{mg} /$ day for 10 days, was added; group 3 were given paracentesis to remove fluid. Fever and constitutional symptoms disappeared faster in group 2 ( $P>0.05)$. After 10 days, there was a significantly greater reduction in the size of pleural effusion in group 2, but after 6 months the difference was not statistically significant. They found corticosteroids and therapeutic paracentesis are not necessary, in the management of TB pleural effusion ${ }^{24}$.

\section{HEMANGIOMA OF THE TRACHEA}

Oral systemic corticosteroids are the mainstay of treatment for problematic hemangiomas; however current information is based on anecdotal experience and retrospective studies

Corticosteroids, both systemic and intralesional have their champions and their skeptic.

In a recent study 20 patients with problematic hemniomas of infancy Pope E et al found that systemic corticosteroids are efficacious in stopping the proliferation of hemangiomas. The oral corticosteroids offered more clinical and biological benefit than the pulse steroids with higher risk of adverse effects ${ }^{25}$.

\section{BRONCHIECTASIS}

Bronchiectasis is a chronic respiratory condition that can present at any age with chronic sputum production or recurrent respiratory infections. Physiotherapy and antibiotics are the cornerstone of therapy and are considered mandatory in long term disease management where symptom control, treatment of exacerbations and the management of any associate 


\section{G.R. Sethi and K.K. Singhal}

respiratory failure are the usual clinical priorities. Inflammation plays a significant role in the pathophysiology of bronchiectasis.

Cochrane review concluded that there are no randomised trials upon which to base recommendations about the use of oral corticosteroids in acute or stable bronchiectasis ${ }^{26}$. Regular use of inhaled corticosteroids, in bronchiectasis, may improve lung function, although available studies were too short and too small to provide any clear evidence to guide pratice.

\section{HYPERPLASIA OF THE THYMUS}

In the unusual situation where an enlarged thymus causes respiratory obstruction, corticosteroids cause a rapid decrease in size of the thymus usually within 5 to days. However, after cessation of corticosteroid therapy, the gland may reach a size greater than that before treatment was initiated. Such a response may also be used to distinguish between a physiological enlargement of the thymus and a neoplasm.

\section{HYPERSENSITIVITY PNEUMONITIS}

Among drug therapies, corticosteroids inhibit the pathologic lesions of HP and usually lead to remission of the acute form of the disease. Steroid therapy is given as prednisolone at the dose of 40 to $60 \mathrm{mg} /$ day, or 1.5 $\mathrm{mg} / \mathrm{kg}$ body weight, in divided doses until there is significant clinical improvement, normalization of the roentgenogram and return of pulmonary function test to normal, at which point the steroid might be slowly tapered over a period of 6 weeks. The long term use of steroid does not alter the respiratory symptoms or lung function in the chronic form of HP.

\section{ARDS}

There is no conclusive evidence to support the use of steroids in ARDS in children. If steroid therapy is used it should probably be for refractory ARDS (e.g. $>7$ days of mechanical ventilation) and the course should be prolonged. Intercurrent infection should be excluded prior to embarking on a prolonged course of corticosteroid therapy.

There is good evidence that in chronic ARDS (persisting beyond 2 weeks) a course of steroids is likely to improve long term survival. Inhaled steroids may also have a role.

\section{ASPIRATION SYNDROMES}

(1) GER and aspiration syndromes: Aspiration of lipid containing liquids such as mineral oils, aromatic oils, or polyethylene glycol solutions provokes an often severe airway obstructive pulmonary inflammatory syndrome. With this form of aspiration, a beneficial response to glucocorticoid therapy is often followed by a long course of glucocorticoid therapy and increase in respiratory distress and a decline in lung function when weaning of steroid therapy is attempted. Steroids have been used for decades in the management of aspiration pneumonia, but there are limited data to support this practice. Studies are generally unsuccessful and sometime the out comes were worse for those treated with coticosteroids ${ }^{27}$.

(2) Foreign body aspiration: Steroids may be indicated if there is significant inflammatory edema or edema caused by endoscopic removal

(3) Meconium aspiration syndromes: There has been considerable interest in the therapeutic potential of steroids in the management of meconium aspiration syndrome, due to their anti-inflammatory properties. The Cochrane review found that at present, there is insufficient evidence to assess the effects of steroid therapy in the management of meconium aspiration syndrome. ${ }^{28}$

(4) Smoke inhalation and carbon monoxide poisoning: Although corticosteroids are frequently advocated in the hope of suppressive inflammation and edema, most controlled studies fail to demonstrate a significant affect.

One review suggests using corticosteroids only for evidence of peripheral airway obstruction, other illnesses requiring steroids, or recent use of steroids.

\section{ATYPICAL PNEUMONIAS}

(1) Hantavirus pulmonary syndrome: Anecdotal reports of corticosteroids suggest that they may be useful.

(2) Mycoplasma pneumoniae: Corticosteroids or immunoglobulins may be of benefit in patients with hemolytic anemia or encephalitis.

(3) Inhalational Anthrax: Corticosteroids have been suggested as adjunctive therapy for its complications such a severe edema, respiratory compromise or meningitis.

\section{DIPHTHERIA}

Intravenous dexamethasone has been given to children with laryngeal diphtheria and airway obstruction, and a small case series suggest that it was beneficial. ${ }^{29}$

\section{PEDIATRIC AIDS}

Although treatment of lymphoid interstitial pneumonia (LIP)/ pulmonary lymphoid hyperplasia (PLH) with 


\section{Pulmonary Diseases and Corticosteroids}

corticosteroid has been reported to improve hypoxemia in a small number of patients, controlled clinical trials have not been carried out.

\section{PEDIATRIC SARS}

SARS is a febrile, respiratory tract illness that is cause by infection with a novel coronavirus (CoV), the SARSassociated $\mathrm{CoV}$ (SARS CoV ). Current treatment is based primarily on supportive care. In an attempt to control inflammation and reduce viral replication, corticosteroids and ribavirin were the most commonly used therapies during the 2003 outbreak. However to date no prospective randomized controlled trials have been conducted to verify the efficacy of any of these treatments, and many safety issues have been raised. Thus not only is the efficacy of steroids unproven in SARS, its timing and dosage regimens are also controversial.

\section{MUSCULAR DYSTROPHIES}

Treatment with prednisolone has been shown to increase muscle strength, and function in DMD patients, but at the expense of too many side effects. Deflazocort, a prednisolone derivative, currently attracts a great deal of interest, since it may have fewer side effects than prednisolone while preserving gross motor and pulmonary functions in DMD patients ${ }^{30}$. However, further randomized trials are necessary to confirm the efficacy and the safety of Deflazocort.

\section{BORDTELLA PERTUSIS}

Uncontrolled observations indicate that corticosteroids and/or $\beta$-adrenergic drugs adjunctive to antibiotics may ameliorate symptoms of Pertusis, but evidence of efficacy is lacking.

\section{REFERENCES}

1. Wapner RJ, Sorokin Y, Thom EA et al. Single versus weekly courses of antenatal corticosteroids: evaluation of safety and efficacy. Am J Obstet Gynae 2006; 195 : 633-642.

2. Crowther CA, Haslam RR, hiller JE et al. Neonatal RDS after repeat exposureof antenatal corticosteroids: a randomized controlled trial. Lancet 2006; 367 : 1913-1919.

3. Halliday HL, Ehrenkranz RA, Doyle LW. Delayed ( $>3$ weeks) postnatal corticosteroids for chronic lung disease in preterm infants. Cochrane Database of Systematic Reviews 2003, Issue 1. Art. No.: CD001145. DOI: 10.1002/ 14651858.CD001145.

4. Doyale JW, Davis PG,Morley CJ et al. Low dose dexamethasone facilitates extubation among chronically ventilator dependent infants: a multicenter, international, randomize, control trial. Pediatrics 2006; 117 : 75-83.

5. O'Shea TM, Washburn LK, Nixon PA et al. Follow up of a randomized, placebo controlled trial of dexamethasone to decrease the duration of ventilator dependency in very low birth weight infants: neurodevelopmental outcomes at 4 to 11 years of age. Pediatrics 2007; 120 : 594-602.

6. Andre $\mathrm{P}$, Theobaud $\mathrm{B}$, vre $\mathrm{MH}$ et al. Mtethylprednisolone, an alternative to dexamethasone in very premature infants at risk of CLD. Intensive Care Medicine 2000; 26 : 1496-500.

7. Watterberg KL, Gerdes GS, Cole GS et al. Prophylaxis of early adrenal insufficiency to prevent bronchopulmonary dysplasia; a multicenteric trial. Pediatrics 2004; 114: 16491657.

8. Global Strategy for Asthma Management and Prevention, Global Initiative for Asthma (GINA) 2007.

9. Edmonds ML, Camargo CA Jr, Brenner BE, Rowe BH. Inhaled steroids for acute asthma following emergency department discharge. Cochrane Database of Systematic Reviews 2000, Issue 3. Art. No.: CD002316. DOI: 10.1002/ 14651858.CD002316

10. Russell K, Wiebe N, Saenz A, Ausejo Segura M, Johnson D, Hartling L, Klassen TP. Glucocorticoids for croup. Cochrane Database of Systematic Reviews 2004, Issue 1. Art. No.: CD001955. DOI: 10.1002/14651858.CD001955.pub2.

11. Patel H, Platt R, Lozano JM, Wang EEL. Glucocorticoids for acute viral bronchiolitis, in infants and young children. Cochrane Database of Systematic Reviews 2004, Issue 3. Art. No.: CD004878. DOI: 10.1002/14651858.CD004878.

12. Blom D, Ermers M, Bont L, van Aalderen WMC, van Woensel JBM. Inhaled corticosteroids during acute bronchiolitis in the prevention of post-bronchiolitic wheezing. Cochrane Database of Systematic Reviews 2007, Issue 1. Art. No.: CD004881. DOI: 10.1002/14651858. CD004881.pub2.

13. Jamaree Teeratakulpisaran, Limwattananon C, Tanupattarachai S et al. Efficacy of Dexamethasone injection for acute bronchiolitis in Hospitalized children. Ped Pulmonol 2007; 42 : 433-439.

14. Corneli HM, Joseph J Zorc, Mahajan P et al. A multicenter, randomized, controlled trial of dexamethasone, for bronchiolitis. N Eng J Med 2007; 357 : 331-339.

15. American Thoracic Society/European Respiratory Society.. Idiopathic pulmonary fibrosis: diagnosis and treatment. International consensus statement. Am J Respir Crit Care Med 2000; $161:$ 646-664.

16. Paramothayan NS, Lasserson TJ, Jones PW. Corticosteroids for pulmonary sarcoidosis. Cochrane Database of Systematic Reviews 2005, Issue 2. Art. No.: CD001114. DOI: 10.1002/ 14651858.CD001114.pub2.

17. Choy EHS, Hoogendijk JE, Lecky B, Winer JB. Immunosuppressant and immunomodulatory treatment, for dermatomyositis and polymyositis. Cochrane Database of Systematic Reviews 2005, Issue 3. Art. No.: CD003643. DOI: 10.1002/14651858.CD003643.pub2

18. Kabra SK, Bhargava S, Lodha R, Satyavani A, Walia M. Idiopathic pulmonary hemosiderosis: clinical profile and follow up of 26 children. Indian Pediatr 2007; 44 : 333-338.

19. Cheng K, Ashby D, Smyth R. Oral steroids for cystic fibrosis. Cochrane Database of Systematic Reviews 1999, Issue 4. Art. No.: CD000407. DOI: 10.1002/14651858. CD000407.

20. Balfour-Lynn I, Walters S, Dezateux C. Inhaled corticosteroids for cystic fibrosis. Cochrane Database of Systematic Reviews 2000, Issue 1. Art. No.: CD001915. DOI: 10.1002/14651858.CD001915.

21. Ian M, Balfour-Lynn, Belinda Lees et al. Multicenter randomized controlled trial of withdrawal of inhaled corticosteroids in cystic fibrosis. Am J Respir Crit Care Med 2006; 173 : 1356-1362. 


\section{G.R. Sethi and K.K. Singhal}

22. Wark PA, Gibson PG, Wilson AJ. Azoles for ABPA associated with asthma. Cochrane Database Syst Rev 2004:CD001108

23. Tokunaga N, Ichikawa Y, Kuboshiro M, Higashi T, Fujino $\mathrm{K}$, Kaji M. A case of miliary tuberculosis associated with acute respiratory failure during corticosteroid treatment of rheumatoid arthritis. Kekkaku, 1990; 65: 539.

24. Mansour AA, Al-Rbeay TB. Adjunct therapy with corticosteroids or paracentesis for treatment of tuberculous pleural effusion. East Mediterr Health J 2006; 12 : 504-508.

25. Pope E, Krafchik BR, Macarthur C et al. Oral versus high dose pulse corticosteroids for problematic infantile hemangiomas: A randomized controlled trial. Pediatrics 2007; 119 : e1239-e1247.

26. Lasserson TJ, Holt K, Milan SJ, Greenstone M.Oral steroids for bronchiectasis (stable and acute exacerbations). Cochrane Database of Systematic Reviews 2001, Issue 4. Art. No.: CD002162. DOI: 10.1002/14651858.CD002162

27. A Peroianni, D Ceccarelli D, Conti V et al. Aspiration pneumonia. Pathophysiological aspects, prevention and management. A review. Panminerva Med 2006; 48 : 231-239.

28. Ward M, Sinn J. Steroid therapy for meconium aspiration syndrome in newborn infants. Cochrane Database of Systematic Reviews 2003, Issue 4. Art. No.: CD003485. DOI: 10.1002/ 14651858.CD003485.

29. Havaldar PG. Dexamethazone in laryngeal diphteritic croup. Ann Troup Pediatrics 1997; 17 : 21-23.

30. Campbel C, Jacob P. Deflazocort treatment of DMD .A systemic review. BMC Neurol 2003; $3: 7$ 